\title{
Topology Fuzzy Normed Algebra
}

\author{
Noori F. Al-Mayahi \\ Suadad M. Abbas \\ Department of Mathematics, College of Computer Science and IT \\ University of Al-Qadisiyah \\ Nfam60@yahoo.com \\ Suadad1987@gmail.com
}

Recived : 24\5\2018

Revised : 30\5\2018

Accepted : $31 \backslash 5 \backslash 2018$

Available online : $\quad \mathbf{5 / 8 / 2 0 1 8}$

DOI: 10.29304/jqcm.2018.10.3.403

\begin{abstract}
:
In this paper, we deal with the basic concepts in topology on fuzzy normed algebra, such as the balls open and balls closed. Next, we study their properties. Furthermore, the concept of derived and closure are discussed.
\end{abstract}

Keywords: Fuzzy field, fuzzy vector space, fuzzy normed space, fuzzy normed algebra .

\section{Mathematics Subject Classification: 46S40 .}

\section{Introduction}

The Theory of Fuzzy sets is introduced by Lotfi Zadeh [1], and the fuzzy topology is defined by Chang [2]. Many mathematicians have tried to extend to fuzzy set theory the main notations of topologies, algebras and groups see ([3][4]) and others as in ([5]-[6]-[7]-[8]-[9]-[10]) .

The apprehensible of fuzzy fields and fuzzy vector spaces was defined first by S.Nanda [11] and redefined by R.Biswas [12]. Also introduced the apprehensible of fuzzy algebra over fuzzy field was defined first S,Nanda [13] and redefined by $\mathrm{Gu}$ and Lu [14]. Gu Wenxiang and $\mathrm{Lu} \mathrm{Tu} \mathrm{[15]} \mathrm{introduced}$ the notions of fuzzy vector spaces. In this papers, we will present new definitions in topology is called a fuzzy normed algebra over fuzzy field. Moreover, some of their characteristics are given in this work. 


\section{Preliminaries:}

In this section, we will recall some definitions which are needed in this work.

\section{Definition (2.1): [15]}

Let $F$ be a field. A fuzzy set $S$ of $F$ is called a fuzzy field of $F$. If the subsequent prerequisites are gratifying :

(1) $S(a+b) \geq \min \{S(a), S(b)\}$, $\forall a, b \in F$.

(2) $S(-a) \geq S(a), \forall a \in F$.

(3) $S(a b) \geq \min \{S(a), S(b)\}$, $\forall a, b \in F$.

(4) $S\left(a^{-1}\right) \geq S(a), \forall a(\neq 0) \in F$.

We dented by $(S, F)$.

\section{Definition (2.2): [15]}

Let $(S, F)$ be a fuzzy field in $F$. A fuzzy set $A$ in a vector space $X$ over $F$ is called fuzzy vector space in $X$ and denoted by $(A, X)$. If the subsequent properties gratifying :

(1) $A(a+b) \geq \min \{A(a), A(b)\}$, $\forall a, b \in X$.

(2) $A(-a) \geq A(a), \forall a \in X$.

(3) $A(\alpha a) \geq \min \{S(\alpha), A(a)\}$, $\forall a \in X$, and $\alpha \in F$.

If $S$ is an usual field then prerequisite (3) above will be fungible by the subsequent axiom :

$A(\alpha a) \geq A(a), \forall \alpha \in F$ and $\forall a \in X$.

\section{Definition (2.3): [14]}

Let $(S, F)$ be a fuzzy field in $F$. A fuzzy set $A$ in algebra $X$ over $F$ is called a fuzzy algebra $(A, X)$ over fuzzy field $(S, F)$. If the subsequent prerequisites materialized :

(1) $A(a+b) \geq \min \{A(a), A(b)\}$, $\forall a, b \in X$.

(2) $A(\alpha a) \geq \min \{S(\alpha), A(a)\}, \forall \alpha \in F$ and $a \in X$.
(3) $A(a b) \geq \min \{A(a), A(b)\}$,

$\forall a, b \in X$.

(4) $S(1) \geq A(a), \forall a \in X$.

\section{Definition (2.4): [16]}

Let $(S, F)$ be a fuzzy field in $F, X$ be vector space over $F$, and let $(A, X)$ be a fuzzy vector space over $(S, F)$. A norm on $(A, X)$ is a function, $\|\cdot\|: X \rightarrow$ $F$ gratifying the subsequent prerequisites:

(1) $S(\|a\|) \geq A(a)$ for all $a \in X$.

(2) $\|a\| \geq 0$ for all $a \in X$.

(3) $\|a\|=0$ if and only if $a=0$.

(4) $\|\alpha a\|=|\alpha|\|a\|$ for all $\alpha \in F$ and $\in X$.

(5) $\|a+b\| \leq\|a\|+\|b\|$ for all $a, b \in X$.

The tuple $(A, X,\|\cdot\|)$ is called a fuzzy normed vector space .

\section{Fuzzy Normed Algebra}

In this section, we will introduced and study the concept of fuzzy normed algebra

\section{Definition (3.1):}

$\operatorname{Let}(S, F)$ be a fuzzy field in $F$, and let $A$ be a fuzzy set in algebra $X$ over $F .(A, X,\|\cdot\|)$ is said to be a fuzzy normed algebra over fuzzy field $(S, F)$ if:

(1) $(A, X)$ is a fuzzy algebra .

(2) $\|\cdot\|$ is a norm on $(A, X)$.

(3) $\|a b\| \leq\|a\|\|b\|$ for all $a, b \in X$.

\section{Definition (3.2):}

Let $(A, X,\|\cdot\|)$ be a fuzzy normed algebra and for each $a_{0} \in X, 0<r$. the open ball $\beta_{r}\left(a_{0}\right)$ in $X$ of radius $r$ and amidst at $a_{0}$ is defined by

$\beta_{r}\left(a_{0}\right)=\left\{a \in X:\left\|a-a_{0}\right\|<r\right.$,

$S\left\|a-a_{0}\right\| \geq \min \left\{A(a), A\left(a_{0}\right)\right\}$

and closed ball $\overline{\beta_{r}}\left(a_{0}\right)$ in $X$ of radius $r$ and amidst at $x_{0}$ is defined by 
$\overline{\beta_{r}}\left(a_{0}\right)=\left\{a \in X:\left\|a-a_{0}\right\| \leq r\right.$,

$S\left\|a-a_{0}\right\| \geq \min \left\{A(a), A\left(a_{0}\right)\right\}$

\section{Theorem (3.3):}

Every open and closed balls in fuzzy normed space are convex .

\section{Proof:}

Let $(A, X,\|\cdot\|)$ be a fuzzy normed algebra .

(1) Let $\mathrm{a}, b \in \beta_{r}\left(a_{0}\right)$ and $0 \leq \alpha \leq 1$

$\Rightarrow\left\|a-a_{0}\right\|<r,\left\|b-a_{0}\right\|<r$.

We must to prove

$$
\begin{aligned}
& \alpha a+(1-\alpha) b \in \beta_{r}\left(a_{0}\right) \\
& \alpha a+(1-\alpha) b-a_{0} \\
& =\alpha\left(a-a_{0}\right)+(1-\alpha)\left(b-a_{0}\right) \\
& \left\|\alpha a+(1-\alpha) b-a_{0}\right\| \\
& =\left\|\alpha\left(a-a_{0}\right)+(1-\alpha)\left(b-a_{0}\right)\right\| \\
& \leq|\alpha|\left\|a-a_{0}\right\|+|1-\alpha|\left\|b-a_{0}\right\| \\
& <\alpha r+(1-\alpha) r=r \text {. }
\end{aligned}
$$

Inasmuch $|1-\alpha|=1-\alpha,|\alpha|=\alpha$

because $\alpha, 1-\alpha \geq 0$.

$$
\begin{aligned}
& \text { And } S\left\|\alpha a+(1-\alpha) b-a_{0}\right\| \\
& \qquad \begin{array}{r}
\geq A\left(\alpha a+(1-\alpha) b-a_{0}\right) \\
=A\left(\alpha a+(1-\alpha) b+\left(-a_{0}\right)\right) \\
\geq \min \left\{A(\alpha a+(1-\alpha) b), A\left(-a_{0}\right)\right\} \\
\geq \min \left\{A(\alpha a+(1-\alpha) b), A\left(a_{0}\right)\right\}
\end{array}
\end{aligned}
$$

(since $A\left(-a_{0}\right) \geq A\left(a_{0}\right)$ )

$\Rightarrow \alpha a+(1-\alpha) b \in \beta_{r}\left(a_{0}\right)$

$\Rightarrow \beta_{r}\left(a_{0}\right)$ is a convex .

(2) Now to prove $\overline{\beta_{r}}\left(a_{0}\right)$ is a convex .

Let $a, b \in \overline{\beta_{r}}\left(a_{0}\right)$ and $0 \leq \alpha \leq 1$

$$
\Rightarrow\left\|a-a_{0}\right\| \leq r,\left\|b-a_{0}\right\| \leq r .
$$

We must to prove

$$
\begin{aligned}
& \alpha a+(1-\alpha) b \in \overline{\beta_{r}}\left(a_{0}\right) \\
& \alpha a+(1-\alpha) b-a_{0} \\
& =\alpha\left(a-a_{0}\right)+(1-\alpha)\left(b-a_{0}\right) \\
& \left\|\alpha a(1-\alpha) b-a_{0}\right\| \\
& =\left\|\alpha\left(b-a_{0}\right)+(1-\alpha)\left(b-a_{0}\right)\right\| \\
& \leq|\alpha|\left\|a-a_{0}\right\|+|1-\alpha|\left\|b-a_{0}\right\|
\end{aligned}
$$

$$
\leq \alpha r+(1-\alpha) r=r .
$$

Inasmuch $|1-\alpha|=1-\alpha,|\alpha|=\alpha$

because , $1-\alpha \geq 0$.

And $S\left\|\alpha a+(1-\alpha) b-a_{0}\right\|$

$$
\begin{aligned}
& \geq A\left(\alpha a+(1-\alpha) b-a_{0}\right) \\
& =A\left(\alpha a+(1-\alpha) b+\left(-a_{0}\right)\right) \\
& \geq \min \left\{A(\alpha a+(1-\alpha) b), A\left(-a_{0}\right)\right\} \\
& \quad \geq \min \left\{A(\alpha a+(1-\alpha) b), A\left(a_{0}\right)\right\}
\end{aligned}
$$

(since $A\left(-a_{0}\right) \geq A\left(a_{0}\right)$ )

$\Rightarrow \alpha a+(1-\alpha) b \in \overline{\beta_{r}}\left(a_{0}\right)$

$\Rightarrow \overline{\beta_{r}}\left(a_{0}\right)$ is a convex .

\section{Definition (3.4):}

Let $(A, X,\|\cdot\|)$ be a fuzzy normed algebra and $B \subseteq$ $X . B$ is said to be an open set in $X$ if for any $a \in B$ there exists $r>0$ such that $\beta_{r}(a) \subset B$. And $B$ is called a closed set in $X$ if $B^{c}$ is an open set in $X$.

\section{Theorem (3.5):}

(1) Each open ball will be an open set.

(2) Each closed ball will be a closed set.

\section{Proof:}

(1)Let $(A, X,\|\cdot\|)$ be a fuzzy normed algebra and let $a_{0} \in X, r>0$ (1) We must to prove $\beta_{r}\left(a_{0}\right)$ is an open set .

Let $a \in \beta_{r}\left(a_{0}\right) \Longrightarrow\left\|a-a_{0}\right\|<r$

$$
\Rightarrow r-\left\|a-a_{0}\right\|>0 \text {. }
$$

But $r_{1}=r-\left\|a-a_{0}\right\| \Rightarrow r_{1}>0$,

we must to prove $\beta_{r_{1}}\left(a_{0}\right) \subset \beta_{r}\left(a_{0}\right)$.

Let $b \in \beta_{r_{1}}\left(a_{0}\right) \Rightarrow\|b-a\|<r_{1}$

$$
\Rightarrow\|b-a\|<r-\left\|a-a_{0}\right\|
$$$$
\Rightarrow\|b-a\|+\left\|a-a_{0}\right\|<r \text {. }
$$

Inasmuch $\left\|b-a_{0}\right\|$

$$
\leq\|b-a\|+\left\|a-a_{0}\right\|
$$

$\Rightarrow\left\|b-a_{0}\right\|<r$.

And $S\left\|b-a_{0}\right\| \geq A\left(b-a_{0}\right)$

$$
\geq \min \left\{A(b), A\left(a_{0}\right)\right\}
$$

$\Rightarrow b \in \beta_{r}\left(a_{0}\right)$ 
$\beta_{r}\left(a_{0}\right)$ is an open set .

(2) We must to prove $\overline{\beta_{r}}\left(a_{0}\right)$ is a closed set. And let $B=\left(\overline{\beta_{r}}\left(a_{0}\right)\right)^{c}$

Inasmuch

$$
\begin{gathered}
\overline{\beta_{r}}\left(a_{0}\right)=\left\{a \in X:\left\|a-a_{0}\right\| \leq r,\right. \\
\left.S\left\|a-a_{0}\right\| \geq \min \left\{A(a), A\left(a_{0}\right)\right\}\right\} \\
\Rightarrow B=\left\{a \in X:\left\|a-a_{0}\right\|>r,\right. \\
\left.S\left\|a-a_{0}\right\|<\min \left\{A(a), A\left(a_{0}\right)\right\}\right\}
\end{gathered}
$$

let $a \in B \Rightarrow\left\|a-a_{0}\right\|>r$.

But $r_{2}=\left\|a-a_{0}\right\|-r \Rightarrow r_{2}>0$,

we must to prove $\beta_{r_{2}}\left(a_{0}\right) \subset B$.

Let $b \in \beta_{r_{2}}\left(a_{0}\right) \Longrightarrow\|b-a\|<r_{2}$

$$
\Rightarrow\|b-a\|<\left\|a-a_{0}\right\|-r
$$

$\Rightarrow\left\|a-a_{0}\right\|-\|b-a\|>r$.

Inasmuch $\left\|a-a_{0}\right\|$

$$
\leq\|a-b\|+\left\|b-a_{0}\right\|
$$

$\Rightarrow\left\|a-a_{0}\right\|-\|b-a\| \leq\left\|b-a_{0}\right\|$

$\Rightarrow\left\|b-a_{0}\right\|>r$.

And $S\left\|b-a_{0}\right\| \geq A\left(b-a_{0}\right)$

$$
\geq \min \left\{A(b), A\left(a_{0}\right)\right\} .
$$

$\Rightarrow b \in B \Rightarrow B_{r_{2}}\left(a_{0}\right) \subset B$

$\Rightarrow B$ is an open set .

Hence $B^{c}=\overline{\beta_{r}}\left(a_{0}\right)$ is a closed set.

\section{Theorem (3.6):}

In any a fuzzy normed algebra $(A, X,\|\cdot\|)$ each single set is a closed and hence finite set is a closed .

\section{proof:}

Let $B$ be a single set.

Suppose $B=\{b\}$, we must to prove $B$ is a closed.

Let $a \in B^{c} \Rightarrow a \neq b$

$$
\Rightarrow\|b-a\|>0 \text {. }
$$

But $r=\|b-a\| \Longrightarrow r>0$.

Since $\|b-a\| \geq r$

$\Rightarrow b \in \beta_{r}(a) \Rightarrow \beta_{r}(a) \cap B=\emptyset$

$\Rightarrow \beta_{r}(a) \subset B^{c} \Rightarrow B^{c}$ is an open set

$\Rightarrow B$ is a closed set .

Now to prove each finite set is a closed .
Let $C$ be a finite subset of $X$ if $C=\emptyset$ the proof ends .

Either if $\neq \varnothing$.

Suppose $C=\left\{c_{1}, c_{2}, \ldots, c_{n}\right\}$

inasmuch $\left\{c_{i}\right\}$ is a closed for each

$i=1,2, \ldots, n$

$\Rightarrow C=\bigcup_{i=1}^{n}\left\{c_{i}\right\}$ is a closed set in $X$.

\section{Definition (3.7):}

Let $(A, X,\|\cdot\|)$ be a fuzzy normed algebra and $\subseteq X$.

(1) The point $a \in X$ is called a limit point to set $B$ if for every $r>0$ there exists

$b \in B$ such that $a \neq b$ and if

$\|a-b\|<r$,

$S\|a-b\| \geq \min \{A(a), A(b)\}$. Set all limit point of set $B$ is called (Derived) of set $B$ and denotes by $B^{\prime}$

$B^{\prime}=\{a \in X: \forall r>0, \exists b \in B \quad \exists b \neq a$, if $\|a-b\|$ $<r$,

$S\|a-b\| \geq \min \{A(a), A(b)\}\}$.

(2) The point $x \in X$ is called a closure point to set $B$ if for all $r>0$ there exist $b \in B$ such that $\|a-b\|<r$,

$S\|a-b\| \geq \min \{A(a), A(b)\}$. The set whose elements all point closure of set $B$ is called (Closure) of set $B$ and denotes by $\bar{B}$ $\bar{B}=\{a \in X: \forall r>0, \exists b \in B$ $\exists\|a-b\|<r$, $S\|a-b\| \geq \min \{A(a), A(b)\}\}$.

\section{Theorem (3.8):}

Let $(A, X,\|\cdot\|)$ a fuzzy normed algebra and let $B \subseteq X$.
(1) $B^{\prime} \subset \bar{B}$.
(2) $\bar{B}=B \cup B^{\prime}$. 


\section{Proof :}

(1)Let $a \in B^{\prime} \Rightarrow$ for all $r>0$ there exists

$b \in B$ such that $b \neq a$ and

$\|a-b\|<r, S\|a-b\| \geq A(a-b)$

$$
\geq \min \{A(a), A(b)\}
$$

$\Rightarrow$ for all $r>0$ there exists $b \in B$ such that $b \neq a$ and $\|a-b\|<r$,

$S\|a-b\| \geq A(a-b)$

$$
\geq \min \{A(a), A(b)\}
$$

$\Rightarrow a \in \bar{B} \Rightarrow B^{\prime} \subset \bar{B}$

(2) From (1) we consider that $B^{\prime} \subset \bar{B}$. This implies that $\cup B^{\prime} \subset B \cup \bar{B}$. But

$B \cup \bar{B}=\bar{B}$ (since $B \subset \bar{B})$ and hence $B \cup B^{\prime} \subset \bar{B}$.

Conversely, suppose $a \in \bar{B}$ there are two possibilities

(a) If $a \in B \Rightarrow a \in B \cup B^{\prime}$

$$
\Rightarrow \bar{B} \subset B \cup B^{\prime}
$$

(b) If $a \notin B$ inasmuch $a \in \bar{B} \Rightarrow$ for all $r>0$ there exists $b \in B$ such that $b \neq a$ and

$$
\begin{aligned}
\|a-b\|<r, S\|a-b\| & \geq A(a-b) \\
& \geq \min \{A(a), A(b)\}
\end{aligned}
$$

inasmuch $b \in B \Rightarrow b \neq a \Rightarrow a \in B^{\prime}$

$\Rightarrow a \in B \cup B^{\prime} \Rightarrow \bar{B} \subset B \cup B^{\prime}$.

Hence $\bar{B}=B \cup B^{\prime}$.

\section{Theorem (3.9):}

Let $B$ convex set in fuzzy normed algebra $(A, X,\|\cdot\|$ ), then $\bar{B}$ convex set .

\section{Proof :}

Let , $b \in \bar{B}, 0 \leq \alpha \leq 1$.

We must to prove that

$\alpha a+(1-\alpha) b \in \bar{B}$.

Inasmuch $a, b \in \bar{B} \Rightarrow a \in \bar{B}$

there exists sequence $\left\{a_{n}\right\}$ in $B$ such that

$a_{n} \rightarrow a$

and $b \in \bar{B}$ there exists sequence $\left\{b_{n}\right\}$ in $B$ such that $b_{n} \rightarrow b$.

Let $z_{n}=\alpha a_{n}+(1-\alpha) b_{n}$.

Inasmuch $a_{n}, b_{n} \in M$ for all $n$

$\Rightarrow \alpha a_{n}+(1-\alpha) b_{n} \in M$

and $a_{n} \rightarrow a, b_{n} \rightarrow b$

$\Rightarrow \alpha a_{n}+(1-\alpha) b_{n} \rightarrow \alpha a+(1-\alpha) b$

$\Rightarrow \alpha a+(1-\alpha) b \in \bar{B}$

$\Rightarrow \bar{B}$ is a convex set .

\section{Theorem (3.10):}

Let $M$ subalgebra of fuzzy normed algebra $(A, X,\|\cdot\|$ ), then $\bar{M}$ subalgebra in $(A, X)$.

\section{Proof:}

Let , $b \in \bar{M}$ and $\mu, \beta \in F$.

We must to prove that $\mu a+\beta b \in \bar{M}$.

Inasmuch $a, b \in \bar{M} \Longrightarrow a \in \bar{M}$ there exists

sequence $\left\{a_{n}\right\}$ in $M$ such that

$a_{n} \rightarrow a$ and $b \in \bar{M}$ there exists

Sequence $\left\{b_{n}\right\}$ in MSuch that

$b_{n} \rightarrow b$.

Let $z_{n}=\mu a_{n}+\beta b_{n}$.

Inasmuch $a_{n}, b_{n} \in M$ for all $n$

$\Longrightarrow \mu a_{n}+\beta b_{n} \in M$

and $a_{n} \rightarrow a, b_{n} \rightarrow b$

$\Rightarrow \mu a_{n}+\beta b_{n} \rightarrow \mu a+\beta b$.

Hence $a+\beta b \in \bar{M}$, then $\bar{M}$ subalgebra

\section{References}

[1] L. A. Zadeh, Fuzzy sets, Information and Control, 8(1965), 1-23.

[2] C. L. Chang, Fuzzy topological space, J. Math. Anal. Appl., 24 (1968), 182-190.

[3] S. Mahmood, Fuzzy Lindelof Closed Spaces and Their Operatios, Journal of General Mathematics Notes, 21(1), (2014), 28-136.

[4] ] P. Bhattacharya and N. P. Mukherjee, Fuzzy relations and fuzzy groups, Inform. Sci.36(1985); $267-282$. 
[5] S. Mahmood, On intuitionistic fuzzy soft bclosed sets in intuitionistic fuzzy soft topological spaces, Annals of Fuzzy Mathematics and Informatics, 10(2), (2015), 221-233.

[6]S. Mahmood and Z. Al-Batat, Intuitionistic Fuzzy Soft LA- Semigroups and Intuitionistic Fuzzy Soft Ideals, International Journal of Applications of Fuzzy Sets and Artificial Intelligence, 6

(2016), 119 - 132.

[7] S. Mahmood, Dissimilarity Fuzzy Soft Points and their Applications, Fuzzy Information and Engineering, 8 (2016), 281-294.

[8]S. Mahmood, New category of the fuzzy dalgebras, Journal of Taibah University for Science,
12(2),
(2018),
143-149.doi:

10.1080/16583655.2018.1451059

[9] N. O. Alshehri, M. Akram and R. S. Al-ghamdi, Applications of Soft Sets in

K-Algebras, Advances in Fuzzy Systems,

ID319542; (2013)8 pages.
[10] P. J. Allen, Construction of many d-algebras, Commun. Korean Math. Soc., 24(2009);361 - 366.

[11] S.Nanada, Fuzzy field and fuzzy linear spaces, Fuzzy Sets and Systems, 19 (1986),89-94.

[12] R.Biswas, Fuzzy fields and fuzzy linear spaces redefined, Fuzzy Sets and Systems 33 (1989) 257259.

[13] S. Nanada, Fuzzy algebras over fuzzy fields, Fuzzy Sets and Systems, 37 (1990), 99-103.

[14] G.Wenxiang and L.Tu, Fuzzy algebras over fuzzy fields redefined, fuzzy sets and systems, 53 (1993), 105-107.

[15] G.Wenxiang and L.Tu, Fuzzy linear space, fuzzy sets and systems, 49 (1992), 377-380.

[16]C. P. Santhosh and T.V.Ramakrishnan ,Norm and inner product on fuzzy linear spaces over fuzzy fields, Iranian Journal of fuzzy systems, 8(2011), pp.135-144.

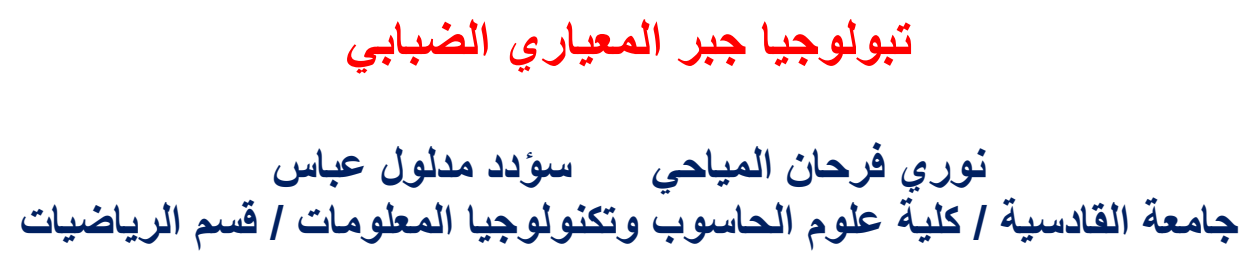

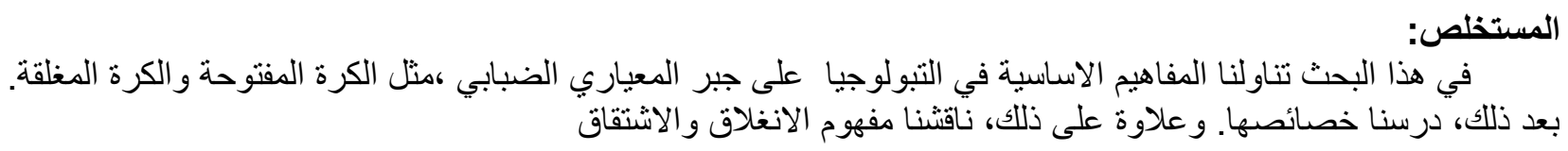

